Pak. j. sci. ind. res. Ser. B: biol. sci. 201255 (3) 122-128

\title{
Diversity Analysis of Marigolds - Tagetes (Asteraceae)
}

\author{
Chithra Santhakumari Amma and Rajalakshmi Radhakrishnan*
}

Department of Botany, University of Kerala, Kariavattom, Thiruvananthapuram- 695581

Kerala , India

(received December 2, 2011; revised September 6, 2012; accepted September 13, 2012)

\begin{abstract}
Characterization of 20 Tagetes cultivars was achieved using morphological and anatomical markers. Tagetes erecta 'Maurel orange' (E4) was the most vigorous compared with other genotypes and produced large flowers with high quality and quantity. An anatomical study of leaf secretory cavities differentiated between the cultivars and revealed that foliar secretory cavities were larger and more abundant in $T$. erecta cultivars than in T. patula cultivars. The size of oil glands also differed in each cultivar. From this study it is concluded that T. erecta cultivars especially T. erecta 'Maurel orange' appeared as more promising one to cultivate and more appropriate to be selected as the parental genotype in the hybridization processes for obtaining new varieties and cultivars. It is concluded that different marker systems (morphological, and anatomical) are appropriate to differentiate between the cultivars of Tagetes.
\end{abstract}

Keywords: marigold, Tagetes erecta, Tagetes patula, morphological traits, cultivars, diversity

\section{Introduction}

Marigolds belong to the family Asteraceae (Compositae), genus Tagetes. Their natural range extends from the southwestern United States into Argentina, with the greatest diversity being in south-central México (Trostle, 1968). The genus Tagetes (Asteraceae) contains 56 species, of which only few species are currently cultivated as horticultural crops. Some companies, release new cultivars every year. Examples are, 'Marvel' line, 'Taishan'line of T. erecta and 'Bonanza' line, 'Boy' line of T.patula which have been widely used in the world (Zhang et al., 2011). Most of the cultivars were produced in the traditional hybridization breeding way (Tian et al., 2007; Wang, 2003, 2009). Besides, some work has also been done on the breeding of transgenic marigold (Charles et al., 2001). Nowadays, the cultivars widely planted throughout the world belong to three species: T. erecta, T.patula and T. tenuifolia. T. erecta flowers are ideal materials for extracting lutein. Therefore, it is very important to study Tagetes plant dut to its great economic value. Many cultivars have been available however, little is known about the differences between them, possibly because many have been introduced in different places with different names as well as different documentation of the original identities.

*Author for correspondence; E-mail: rajalakshmi.murali @gmail.com
Plant taxonomy has been mainly based upon morphological, cytological, and molecular biological analysis, etc. Morphological characters, both qualitative and quantitative, have long been used to identify species, varieties and cultivars to evaluate relationships, and discriminate between varieties and cultivars. Morphological traits continue to be the first step in the studies of genetic relationships in most breeding programmes (Van Beuningen and Busch, 1997; Cox and Murphy, 1990). In Tagetes breeding programmes, the major emphasis has been on the collection and conservation of genetic pools. There are numerous cultivars that cover a wide spectrum of growth habits, floral traits, environmental responses and varying pest and disease susceptibilities. So a wide range of characters have to be considered to select a superior germplasm that serves as the essential foundation for the breeding of new improved varieties as well as cultivars. Earlier studies on Tagetes cultivars using morphological traits such as plant habit, leaf traits, flower size, flower colour and pigmentation are rare. Anatomical characters for the identification of oil glands are more important to characterize the presence of essential oil in leaves. These traits are found to be of great importance to distinguish genetic variability, and can lead to a better classification of Tagetes cultivars. Thus, the objectives of the present study were to analyze the diversity of 20 cultivars belonging to two species of Tagetes (T.erecta and T. patula) and to classify them into particular groups 
based on morphological and anatomical variation. It can be used for cultivar identification and further use in crop improvement through breeding programmes.

\section{Materials and Methods}

Plant material. Twelve cultivars of $T$. erecta and eight cultivars of $T$. patula were procured from nurseries for this study. 20 genotypes were grown at the Botany Department Garden, Kariavattom, Thiruvananthapuram (Kerala). All plants were replicated by stem cuttings and these vegetative propagated plants (clones) were used for further study. Crop management was done according to recommended agronomic practices.

Propagation. Clones were produced through stem cuttings. Plants were grown quickly and produce flowers. For seed germination seeds were sown in pots (germination percentage was only $50 \%$ ). Out of twenty cultivars only twelve were germinated and rest of it was not being germinated at a stipulated period. The seeds were germinated in 3-5 days.

Morphological study. Twenty-cultivars belonging to two species of Tagetes were selected for this study. To avoid phenological differences between individuals, plants that had started to bloom were chosen for the study of vegetative traits and flowers were collected at the anthesis stage. For the study of leaf traits, the third leaf from the apex was chosen. Thirty morphological characters; 20 qualitative and 10 quantitative characters (Table 1), were considerd. Five plants were measured for each trait.

The terminology of Hickey and King (2000) was adopted to describe the qualitative characters. Morphological and floral traits were studied with the help of hand lens. Photographs were taken with a digital camera (DP11, Olympus, Tokyo, Japan). A datasheet was designed, and information was recorded for 10 quantitative and 20 qualitative characters (Table 2-3). To evaluate significant differences in quantitative traits, one-way ANOVA was performed. A correlation based PCA was performed to select taxonomically significant qualitative and quantitative characters and to detect outliers. The relative taxonomic distance between the cultivars was calculated by cluster analysis. Pair-wise relationships were estimated by the Euclidean distance coefficient. The distance matrix was represented as a phenogram by the UPGMA clustering method (Sneath and Sokal, 1973). All statistical tests except ANOVA were performed with the MultiVariate Statistical Package
Table 1. Morphological characters of Tagetes taken for the study

\begin{tabular}{ll}
\hline \hline Parameters & \\
Qualitative characters & Observation \\
\hline Stem surface & 1. Ribbed; 2. Ribbed and Hairy; \\
& 3. Stem Reddish and Ribbed \\
Petiole & 1.Short; 2. Long \\
Leaf position & 1. Opposite; 2. Alternate \\
Leaf Shape & 1.Lanceolate; 2.Ovate; 3.Acute \\
Leaf apex & 1.Acute; 2. Ovate \\
Leaf base & 1.Acute; 2. Ovate \\
Leaf margin & 1.Serrate; 2. Non serrate \\
Leaf colour & 1. Green; 2. Not green \\
Lamina symmetry & 1. Symmetry; 2. Non symmetry \\
Head type & 1. Heterozygous; 2. Homozygous \\
Inflorescence type & 1. Corymbose; 2. Not corymbose \\
Inflorescenceposition & 1. Solitary; 2. Axillary \\
Involucre type & 1. Uni serrate; 2. Multi serrate \\
Pappus type & 1. Awns; 2. Capillary; 3. Bristles \\
Style colour & 1.Yellow; 2. Orange; 3. Pale \\
Stigma colour & yellow 4. Pale orange \\
Stigma type & 1.Yellow; 2. Orange; 3. Pale \\
Quantitative traits & yellow 4. Pale orange \\
Leaf length & 1. Bifid; 2.Not Bifid \\
Leaf breadth & $\mathrm{cm}$ \\
Leaf area & $\mathrm{cm}$ \\
Leaf perimeter & $\mathrm{cm}$ 2 \\
Peduncle length & $\mathrm{cm}$ \\
Internode length & $\mathrm{cm}$ \\
Involucre length & $\mathrm{cm}$ \\
Ovary length & $\mathrm{mm}$ \\
Style length & \\
\hline
\end{tabular}

(MVSP) version 3.1 (Kovach Computing Services, Wales, UK). ANOVA was carried out with SPSS 7.5 (SPSS, 1999).

Anatomical study. For the study of some anatomical traits, morphologically distinct accessions were selected based on the UPGMA cluster analysis, PCA and oneway ANOVA. They were $T$. erecta 'Indian orange'(E1), T. erecta 'Maurel orange' (E4), T. erecta 'Antigua white (E6), T. erecta 'Discovery yellow' (E7), T. erecta 'Antigua Orange' (E9), T. erecta ' Safari Tangerine' (E11), T. patula 'Double Eagle' (P1), T. patula 'Double Orange' (P5), T. patula 'Inca Cream'(P6) and T. patula 'Safari Yellow' (P8). For the leaf anatomy analysis, fully developed leaves were collected and fixed in ethanol. Transverse sections of the midrib, as well as 
Table 2. Variations in quantitative characters of Tagetes

\begin{tabular}{|c|c|c|c|c|c|c|c|c|c|c|c|}
\hline \multirow[b]{2}{*}{ Name } & \multicolumn{10}{|c|}{ Morphological Characters } & \multirow[b]{2}{*}{$\begin{array}{l}\text { Style } \\
\text { length } \\
(\mathrm{cm})\end{array}$} \\
\hline & $\begin{array}{l}\text { Cultivar } \\
\text { code }\end{array}$ & $\begin{array}{l}\text { Leaf } \\
\text { length } \\
(\mathrm{cm})\end{array}$ & $\begin{array}{l}\text { Leaf } \\
\text { breadth } \\
(\mathrm{cm})\end{array}$ & $\begin{array}{l}\text { Leaf } \\
\text { area } \\
\left(\mathrm{cm}^{2}\right)\end{array}$ & $\begin{array}{l}\text { Leaf } \\
\text { perimeter } \\
(\mathrm{cm})\end{array}$ & $\begin{array}{l}\text { Peduncle } \\
\text { length } \\
(\mathrm{cm})\end{array}$ & $\begin{array}{l}\text { Intermodal } \\
\text { length } \\
(\mathrm{cm})\end{array}$ & $\begin{array}{l}\text { Involucres } \\
\text { length } \\
(\mathrm{cm})\end{array}$ & $\begin{array}{l}\text { Pappus } \\
\text { length } \\
(\mathrm{mm})\end{array}$ & $\begin{array}{l}\text { Ovary } \\
\text { length } \\
(\mathrm{mm})\end{array}$ & \\
\hline T. erecta'Indian Orange' & E1 & $6.2 \pm 0.08$ & $1.6 \pm-0.04$ & $7.3 \pm-0.04$ & $9.2 \pm-0.1$ & $6.3 \pm-0.06$ & $1.3 \pm-0.04$ & $3.2 \pm-0.07$ & $2.2 \pm-0.1$ & $1.4 \pm-0.04$ & $2.1 \pm-0.06$ \\
\hline T. erecta'Atlantis Orange' & E2 & $6.3 \pm 0.06$ & $1.6 \pm-0.04$ & $7.2 \pm-0.04$ & $9.3 \pm-0$ & $6.1 \pm-0.07$ & $1.2 \pm-0.04$ & $3.1 \pm-0.04$ & $2.2 \pm-0.05$ & $1.2 \pm-0.08$ & $2.1 \pm-0.04$ \\
\hline T. erecta'Antigua Yellow' & E3 & $6.5 \pm 0.07$ & $1.5 \pm-0.04$ & $7.3 \pm-0.04$ & $9.4 \pm-0.9$ & $6.2 \pm-0.08$ & $1.4 \pm-0.04$ & $3.1 \pm-0.04$ & $2.6 \pm-0.09$ & $1.2 \pm-0.04$ & $2.2 \pm-0.04$ \\
\hline T. erecta'Maurel Orange' & E4 & $6.4 \pm 0.08$ & $1.6 \pm-0.04$ & $7.2 \pm-0.85$ & $8.2 \pm-0.1$ & $5.5 \pm-0.15$ & $1.2 \pm-0.04$ & $3.1 \pm-0.06$ & $2.3 \pm-0.06$ & $1.1 \pm-0.07$ & $2.1 \pm-0.06$ \\
\hline T. erecta'Maurel Yellow' & E5 & $6.4 \pm 0.05$ & $1.5 \pm-0.06$ & $7.1 \pm-0.08$ & $9.2 \pm-0.08$ & $6.3 \pm-0.08$ & $1.4 \pm-0.08$ & $3.1 \pm-0.08$ & $2.6 \pm-0.04$ & $1.2 \pm-0.04$ & $2.3 \pm-0.10$ \\
\hline T. erecta'Antigua White' & E6 & $6.3 \pm 0.04$ & $1.4 \pm-0.04$ & $7.2 \pm-0.6$ & $9.1 \pm-0.6$ & $6.4 \pm-0.1$ & $1.3 \pm-0.04$ & $3.1 \pm-0.08$ & $2.1 \pm-0.06$ & $1.4 \pm-0.10$ & $2.1 \pm-0.07$ \\
\hline T. erecta 'Discovery Yellow' & E7 & $6.5 \pm 0.04$ & $1.6 \pm-0.06$ & $7.3 \pm-0.6$ & $9.1 \pm-0.7$ & $6.3 \pm-0.06$ & $1.2 \pm-0.06$ & $3.1 \pm-0.06$ & $2.6 \pm-0.04$ & $1.3 \pm-0.04$ & $2.2 \pm-0.09$ \\
\hline T. erecta'Sweet White' & E8 & $6.0 \pm 0.06$ & $1.5 \pm-0.04$ & $7.3 \pm-0$ & $9.3 \pm-0.06$ & $6.3 \pm-0.08$ & $1.2 \pm-0.09$ & $3.2 \pm-0.06$ & $2.6 \pm-0.06$ & $1.1 \pm-0.07$ & $2.2 \pm-0.09$ \\
\hline T. erecta'Antigua Orange' & E9 & $6.6 \pm 0.04$ & $1.6 \pm-0.06$ & $7.2 \pm-0.6$ & $9.3 \pm-0.04$ & $6.3 \pm-0.06$ & $1.4 \pm-0.10$ & $3.2 \pm-0.06$ & $2.4 \pm-0.04$ & $1.2 \pm-0.02$ & $2.1 \pm-0.04$ \\
\hline T. erecta'Inca Yellow' & E10 & $6.4 \pm 0.06$ & $1.3 \pm-0.04$ & $7.4 \pm-0.08$ & $9.4 \pm-0.08$ & $6.65 \pm-0.1$ & $1.5 \pm-0.08$ & $3.1 \pm-0.06$ & $2.4 \pm-0.04$ & $1.4 \pm-0.06$ & $2.1 \pm-0.06$ \\
\hline T. erecta' Safari Tangerine' & E11 & $6.4 \pm 0.07$ & $1.2 \pm-0.07$ & $7.2 \pm-0.7$ & $9.2 \pm-0.06$ & $6.5 \pm-0.1$ & $1.4 \pm-0.06$ & $3.4 \pm-0.1$ & $2.7 \pm-0.01$ & $1.3 \pm-0.04$ & $2.1 \pm-0.04$ \\
\hline T. erecta' Sweet Cream’ & E12 & $6.6 \pm 0.06$ & $1.4 \pm-0.04$ & $7.4 \pm-0.04$ & $9.4 \pm-0.1$ & $6.3 \pm-0.07$ & $1.2 \pm-0.10$ & $3.3 \pm-0.08$ & $2.5 \pm-0.04$ & $1.3 \pm-0.06$ & $2.2 \pm-0.0$ \\
\hline T. patula 'Double Eagle' & P1 & $5.3 \pm 0.07$ & $1.2 \pm-0.08$ & $6.9 \pm-0.07$ & $8.3 \pm-0.08$ & $5.0 \pm-0.14$ & $1.2 \pm-0.00$ & $2.1 \pm-0.04$ & $3.0 \pm-0.08$ & $2.1 \pm-0.06$ & $2.1 \pm-0.06$ \\
\hline T. patula'Inca Orange' & P2 & $5.4 \pm 0.08$ & $1.2 \pm-0.08$ & $7.1 \pm-0.06$ & $8.3 \pm-0.07$ & $5.3 \pm-0.08$ & $1.2 \pm-0.07$ & $2.3 \pm-0.04$ & $3.1 \pm-0.06$ & $2.2 \pm-0.09$ & $2.2 \pm-0.09$ \\
\hline T. patula 'Orange Eagle' & P3 & $5.3 \pm 0.08$ & $1.3 \pm-0.06$ & $7.1 \pm-0.06$ & $8.2 \pm-0.10$ & $5.4 \pm-0.09$ & $1.2 \pm-0.08$ & $2.1 \pm-0.06$ & $3.2 \pm-0.06$ & $1.1 \pm-0.04$ & $2.1 \pm-0.04$ \\
\hline T. patula 'Safari' & P4 & $5.4 \pm 0.08$ & $1.4 \pm-0.04$ & $7.2 \pm-0.02$ & $8.2 \pm-0.09$ & $5.6 \pm-0.06$ & $1.2 \pm-0.04$ & $3.2 \pm-0.00$ & $2.1 \pm-0.06$ & $1.3 \pm-0.06$ & $1.3 \pm-0.06$ \\
\hline T. patula 'Double Orange' & P5 & $5.2 \pm 0.04$ & $1.1 \pm-0.06$ & $6.9 \pm-0.04$ & $8.4 \pm-0.11$ & $5.6 \pm-0.06$ & $1.3 \pm-0.04$ & $3.2 \pm-0.01$ & $2.1 \pm-0.06$ & $1.2 \pm-0.07$ & $1.2 \pm-7.07$ \\
\hline T. patula 'Inca Cream' & P6 & $5.7 \pm 0.05$ & $1.3 \pm-0.06$ & $6.9 \pm-0.04$ & $8.3 \pm-0.06$ & $5.3 \pm-0.00$ & $1.2 \pm-0.08$ & $3.0 \pm-0.06$ & $2.4 \pm-0.02$ & $1.1 \pm-0.0$ & $1.1 \pm-0.08$ \\
\hline T. patula 'Mesa Gold' & P7 & $5.3 \pm 0.09$ & $1.0 \pm-0.06$ & $7.0 \pm-0.08$ & $8.5 \pm-0.07$ & $5.3 \pm-0.09$ & $1.2 \pm-0.08$ & $3.0 \pm-7.5$ & $2.2 \pm-0.08$ & $1.1 \pm-0.06$ & $1.1 \pm-6.29$ \\
\hline T. patula'Safari Yellow' & P8 & $5.2 \pm-0.08$ & $1.3 \pm-0.02$ & $6.7 \pm-0.06$ & $8.4 \pm-0.08$ & $5.6 \pm-0.04$ & $1.2 \pm-0.08$ & $3.1 \pm-0.08$ & $2.1 \pm-0.06$ & $1.1 \pm-0.06$ & $1.1 \pm-6.29$ \\
\hline
\end{tabular}

Table 3. Qualitative characters of Tagetes

Cultivars

\begin{tabular}{|c|c|c|c|c|c|c|c|c|c|c|c|c|c|c|c|c|c|c|c|c|}
\hline Characters & E1 & E2 & E3 & E4 & E5 & E6 & E7 & E8 & E9 & E10 & E11 & E12 & P1 & P2 & P3 & P4 & P5 & P6 & P7 & P8 \\
\hline Habit & $\mathrm{H}$ & $\mathrm{H}$ & $\mathrm{H}$ & $\mathrm{H}$ & $\mathrm{H}$ & $\mathrm{H}$ & $\mathrm{H}$ & $\mathrm{H}$ & $\mathrm{H}$ & $\mathrm{H}$ & $\mathrm{H}$ & $\mathrm{H}$ & $\mathrm{H}$ & $\mathrm{H}$ & $\mathrm{H}$ & $\mathrm{H}$ & $\mathrm{H}$ & $\mathrm{H}$ & $\mathrm{H}$ & $\mathrm{H}$ \\
\hline Stem surface & $\mathrm{RH}$ & $\mathrm{R}$ & $\mathrm{R}$ & RH & RH & RH & RH & $\mathrm{RH}$ & $\mathrm{R}$ & $\mathrm{R}$ & $\mathrm{RR}$ & $\mathrm{RH}$ & $\mathrm{R}$ & $\mathrm{RR}$ & RH & $\mathrm{RR}$ & $\mathrm{R}$ & $\mathrm{RR}$ & $\mathrm{RR}$ & $\mathrm{RR}$ \\
\hline Branching nature & SB & PB & NB & $\mathrm{PB}$ & NB & NB & SB & SB & SB & PB & $\mathrm{PB}$ & $\mathrm{PB}$ & B & B & PB & $\mathrm{B}$ & PB & PB & PB & PB \\
\hline Petiole & $\mathrm{L}$ & $\mathrm{L}$ & $\mathrm{L}$ & $\mathrm{L}$ & $\mathrm{L}$ & $\mathrm{L}$ & $\mathrm{L}$ & $\mathrm{L}$ & $\mathrm{L}$ & $\mathrm{L}$ & $\mathrm{L}$ & $\mathrm{L}$ & S & M & $\mathrm{S}$ & S & S & S & S & S \\
\hline Leaf position & OP & OP & $\mathrm{AL}$ & OP & $\mathrm{AL}$ & $\mathrm{AL}$ & OP & OP & $\mathrm{AL}$ & OP & $\mathrm{AL}$ & OP & OP & OP & $\mathrm{AL}$ & $\mathrm{AL}$ & $\mathrm{AL}$ & $\mathrm{AL}$ & OP & OP \\
\hline Leaf shape & $\mathrm{OV}$ & LA & LA & LA & $\mathrm{OV}$ & $\mathrm{AC}$ & $\mathrm{AC}$ & $\mathrm{AC}$ & $\mathrm{AC}$ & $\mathrm{AC}$ & $\mathrm{AC}$ & $\mathrm{AC}$ & LA & $\mathrm{OV}$ & $\mathrm{OV}$ & OV & $\mathrm{OV}$ & $\mathrm{OV}$ & $\mathrm{AC}$ & $\mathrm{OV}$ \\
\hline Leaf apex & $\mathrm{AC}$ & $\mathrm{AC}$ & $\mathrm{AC}$ & $\mathrm{AC}$ & $\mathrm{AC}$ & OV & $\mathrm{OV}$ & $\mathrm{OV}$ & OV & OV & $\mathrm{OV}$ & $\mathrm{OV}$ & $\mathrm{AC}$ & $\mathrm{AC}$ & $\mathrm{AC}$ & $\mathrm{AC}$ & $\mathrm{AC}$ & $\mathrm{AC}$ & OV & $\mathrm{AC}$ \\
\hline Leaf base & $\mathrm{AC}$ & $\mathrm{AC}$ & $\mathrm{AC}$ & OV & $\mathrm{AC}$ & OV & $\mathrm{AC}$ & $\mathrm{OV}$ & OV & OV & $\mathrm{AC}$ & $\mathrm{AC}$ & OV & $\mathrm{AC}$ & OV & $\mathrm{AC}$ & $\mathrm{AC}$ & $\mathrm{AC}$ & $\mathrm{OV}$ & $\mathrm{AC}$ \\
\hline Leaf margin & $\mathrm{SE}$ & SE & SE & $\mathrm{SE}$ & SE & $\mathrm{SE}$ & SE & SE & $\mathrm{SE}$ & SE & SE & $\mathrm{SE}$ & $\mathrm{SE}$ & SE & SE & $\mathrm{SE}$ & $\mathrm{SE}$ & SE & $\mathrm{SE}$ & SE \\
\hline Leaf colour & G & G & G & G & G & G & G & G & G & G & G & G & G & G & G & G & G & G & G & G \\
\hline Lamina symmetry & SY & SY & SY & SY & SY & SY & SY & SY & SY & SY & SY & SY & SY & SY & SY & SY & SY & SY & SY & SY \\
\hline Flower colour & $\mathrm{O}$ & LO & PY & DO & FY & WH & DY & $\mathrm{CW}$ & $\mathrm{PO}$ & LY & YO & $\mathrm{C}$ & YR & $\mathrm{YO}$ & DO & DY & DR & $\mathrm{YC}$ & GY & DY \\
\hline Head type & $\mathrm{HO}$ & $\mathrm{HO}$ & $\mathrm{HO}$ & $\mathrm{HO}$ & $\mathrm{HO}$ & $\mathrm{HO}$ & $\mathrm{HO}$ & $\mathrm{HO}$ & $\mathrm{HO}$ & $\mathrm{HO}$ & $\mathrm{HO}$ & $\mathrm{HO}$ & $\mathrm{HE}$ & $\mathrm{HE}$ & $\mathrm{HE}$ & $\mathrm{HE}$ & $\mathrm{HE}$ & $\mathrm{HE}$ & $\mathrm{HE}$ & $\mathrm{HE}$ \\
\hline Inflorescence type & $\mathrm{CO}$ & $\mathrm{CO}$ & $\mathrm{CO}$ & $\mathrm{CO}$ & $\mathrm{CO}$ & $\mathrm{CO}$ & $\mathrm{CO}$ & $\mathrm{CO}$ & $\mathrm{CO}$ & $\mathrm{CO}$ & $\mathrm{CO}$ & $\mathrm{CO}$ & $\mathrm{CO}$ & $\mathrm{CO}$ & $\mathrm{CO}$ & $\mathrm{CO}$ & $\mathrm{CO}$ & $\mathrm{CO}$ & $\mathrm{CO}$ & $\mathrm{CO}$ \\
\hline Inflorescence position & $\mathrm{AX}$ & $\mathrm{AX}$ & SO & $\mathrm{AX}$ & $\mathrm{AX}$ & SO & SO & SO & $\mathrm{AX}$ & $\mathrm{AX}$ & $\mathrm{SO}$ & SO & SO & SO & SO & $\mathrm{SO}$ & $\mathrm{AX}$ & $\mathrm{CO}$ & $\mathrm{CO}$ & $\mathrm{CO}$ \\
\hline Involucre type & UN & UN & UN & UN & UN & UN & UN & UN & UN & UN & UN & UN & MU & MU & MU & MU & MU & MU & MU & MU \\
\hline Pappus type & $\mathrm{CB}$ & CB & CB & $\mathrm{CB}$ & $\mathrm{CB}$ & $\mathrm{CB}$ & $\mathrm{CB}$ & $\mathrm{CB}$ & $\mathrm{CB}$ & $\mathrm{CB}$ & $\mathrm{CB}$ & $\mathrm{CB}$ & AW & AW & AW & AW & AW & AW & AW & AW \\
\hline Style colour & y & PY & $\mathrm{Y}$ & $\mathrm{PO}$ & Y & $\mathrm{O}$ & WH & $\mathrm{O}$ & WH & $\mathrm{O}$ & Y & $\mathrm{O}$ & Y & $\mathrm{O}$ & PY & $\mathrm{O}$ & $\mathrm{PO}$ & $\mathrm{PO}$ & $\mathrm{Y}$ & $\mathrm{O}$ \\
\hline Stigma colour & Y & $\mathrm{Y}$ & Y & $\mathrm{PO}$ & $\mathrm{Y}$ & $\mathrm{O}$ & WH & $\mathrm{O}$ & WH & $\mathrm{O}$ & Y & $\mathrm{YO}$ & Y & $\mathrm{O}$ & PY & $\mathrm{O}$ & PO & $\mathrm{PO}$ & $\mathrm{Y}$ & $\mathrm{O}$ \\
\hline Stigma type & $\mathrm{BF}$ & $\mathrm{BF}$ & $\mathrm{BF}$ & $\mathrm{BF}$ & $\mathrm{BF}$ & $\mathrm{BF}$ & $\mathrm{BF}$ & $\mathrm{BF}$ & $\mathrm{BF}$ & $\mathrm{BF}$ & $\mathrm{BF}$ & $\mathrm{BF}$ & $\mathrm{BF}$ & $\mathrm{BF}$ & $\mathrm{BF}$ & $\mathrm{BF}$ & $\mathrm{BF}$ & $\mathrm{BF}$ & $\mathrm{BF}$ & $\mathrm{BF}$ \\
\hline
\end{tabular}

$\mathrm{R}=$ Ribbed; $\mathrm{RH}=$ Ribbed and Hairy; $\mathrm{RR}=$ Stem Reddish and Ribbed; $\mathrm{SB}=$ Sparingly branched; $\mathrm{PB}=$ Profusely branched; NB $=$ Not branched; $\mathrm{S}=$ Short; $\mathrm{L}=$ Long; $\mathrm{OP}=$ Opposite; $\mathrm{AL}=$ Alternate; $\mathrm{LA}=$ Lanceolate; $\mathrm{OV}=$ Ovate; $\mathrm{AC}=$ Acute; $\mathrm{S}=\mathrm{Serate} ; \mathrm{G}=\mathrm{Green} ; \mathrm{S}=$ Symmetry; $\mathrm{HE}=$ Heterozygous; $\mathrm{HO}=$ Homozygous; $\mathrm{C}=$ Corymbose; $\mathrm{SO}=$ Solitary; $\mathrm{AX}=$ Axillary; $\mathrm{UN}=$ Uni serrate; $\mathrm{MU}=\mathrm{Multi}$ serrate; $\mathrm{AW}=$ Awns $\mathrm{CB}=$ Capillary bristles; $\mathrm{Y}=$ Yellow; $\mathrm{O}=$ Orange; $\mathrm{PY}=$ Pale yellow; $\mathrm{PO}=$ Pale orange; $\mathrm{BF}=\mathrm{Bifid}$. 
transverse sections of the leaf-blade in the intercostal region and in the margin were obtained. Hand-sections were performed using a razor blade, cleared with sodium hypochlorite, washed with water, stained with astrablausafranin (Bukatsch, 1972) and mounted in 50\% glycerol. Anatomical features were studied by using a stereo zoom microscope (SZ61, Olympus, Tokyo, Japan). Photographs were taken with a digital camera (DP11, Olympus, Tokyo, Japan) attached to the microscope.

\section{Results and Discussion}

Morphological study. Morphological characters. The plants were herbs and the shoot length of T.erecta ranged between 12 to $16 \mathrm{~cm}$, while the shoot length of T. patula ranged between 8 to $10 \mathrm{~cm}$. Stem colour was reddish or green and stem surface was ribbed or ribbed and hairy. Some genotypes were unbranched as E3, E5 and E6 while the other genotypes were sparingly or profusely branched. Internodal length varied from 1.2 to $1.5 \mathrm{~cm}$ in T. erecta and 1.2 to $1.3 \mathrm{~cm}$ in T. patula. The leaves were green, compound and symmetrical, lanceolate, acute or ovate with serrate margin. Leaves were opposite or alternate. Leaf length ranged from 6.2 to $6.6 \mathrm{~cm}$ in T. erecta and 5.2 to $5.7 \mathrm{~cm}$ in T. patula. Leaf width ranged between 1.2 to $1.6 \mathrm{~cm}$ in $T$. erecta and 1.0 to $1.4 \mathrm{~cm}$ in T. patula. Leaf area and perimeter in T. erecta ranged between 7.1 to $7.4 \mathrm{~cm}^{2}$ and 8.2 to $9.4 \mathrm{~cm}$ respectively. In T. patula it ranged between 6.7 to 7.2 $\mathrm{cm}^{2}$ and 8.2 to $8.5 \mathrm{~cm}$ respectively.

The inflorescence consists of numerous ray and disc florets in head or capitulum. Head type was homogamous in T.erecta but herterogamous in T. patula. Variations could be noted in the flower colour. It was orange in E1, E2, E4 and E9. Various yellowish shades were seen in E3, E5, E7, E10 and E11. White, creamy white and cream colours were found in E6, E8 and E12 respectively. Some cultivars of T.patula showed distinct colours in outside and inside of the head inflorescence. Reddish yellow outside and yellowish inside in P1, orange outside and yellow inside in P3 and whitish cream outside and yellow inside in P6. Dark red coloured ray florets were found in P5. Involucre type in T.erecta was uniserrate but in $T$. patula it was multi serrate. Capillary bristles were found in T. erecta but awns in $T$. patula. Involucre length of T. erecta ranged between 3.1 to $3.4 \mathrm{~cm}$ where as in T. patula it ranged between 2.1 to $3.2 \mathrm{~cm}$. Pappus length of flowers ranged between 2.1 to $2.7 \mathrm{~cm}$ in $T$. erecta and 2.1 to $3.2 \mathrm{~cm}$ in T. patula. Ovary and style length of T. patula were found to be more variable compared to T. erecta. In T. patula ovary length ranged between 1.1 to $2.2 \mathrm{~cm}$ where as in T. erecta it was 1.1 to $1.4 \mathrm{~cm}$. Style length ranged between 1.1 to $2.2 \mathrm{~cm}$ in T. patula where as in T. erecta it was 2.1 to $2.3 \mathrm{~cm}$. Stigma was bifid in both species.

Morphometric analysis. ANOVA. All the quantitative characters were found to be significant.

PCA. Qualitative traits. In the PCA of qualitative data of T.erecta, $56.8 \%$ of the phenetic variance was accounted for by the first principal axis, followed by $17.3 \%$ for the second, $8.2 \%$ for the third, $5.5 \%$ for the fourth and $5.3 \%$ for the fifth principal component (Table 4). Most of the selected qualitative traits were found principally influential in the PCA. Branching nature, leaf morphology and flower colour were found influential in the most variable first principal component.

In the PCA of qualitative data of T.patula, $38.6 \%$ of the phenetic variance was accounted for by the first principal axis, followed by $25.5 \%$ for the second, $16.2 \%$ for the third, $8.6 \%$ for the fourth and $6.9 \%$ for the fifth principal component (Table 4). Most of the selected qualitative traits were found principally influential in the PCA. Leaf position, leaf morphology and flower colour were found influential in the most variable first principal component.

Quantitative traits. In the PCA of quantitative data of T.erecta, $43.3 \%$ of the phenetic variance was accounted for by the first principal axis, followed by $23.2 \%$ for the second, $15.8 \%$ for the third and $10.3 \%$ for the fourth principal component (Table 5). The first principal component accounted for $62.7 \%$ of phenotypic variance and the second component for $21.1 \%$ (Table 5) in T.patula. All the quantitative traits except leaf characters were found to have significant loadings in PCA.

Cluster analysis. The UPGMA phenogram based on the morphological characters shows two main principal clusters (Fig. 1) separating the two species. In the T.patula cluster, the cultivars $\mathrm{P} 3$ and $\mathrm{P} 4$ are separated out with others, and again P2 and P6 are separated out. Cultivar P7 is more distinct from P2, P6, P3 and P4 but more close to P8, P1 and P5. In the T. erecta cluster cultivar E4 is more distant from others. E8 and E7 as well as E10 and E11 are more close to each other.

Anatomical study. Anatomical studies revealed that foliar secretory cavities were more abundant in T. erecta cultivars than in $T$. patula cultivars. The secretory structures were found in the lamina between the palisade 


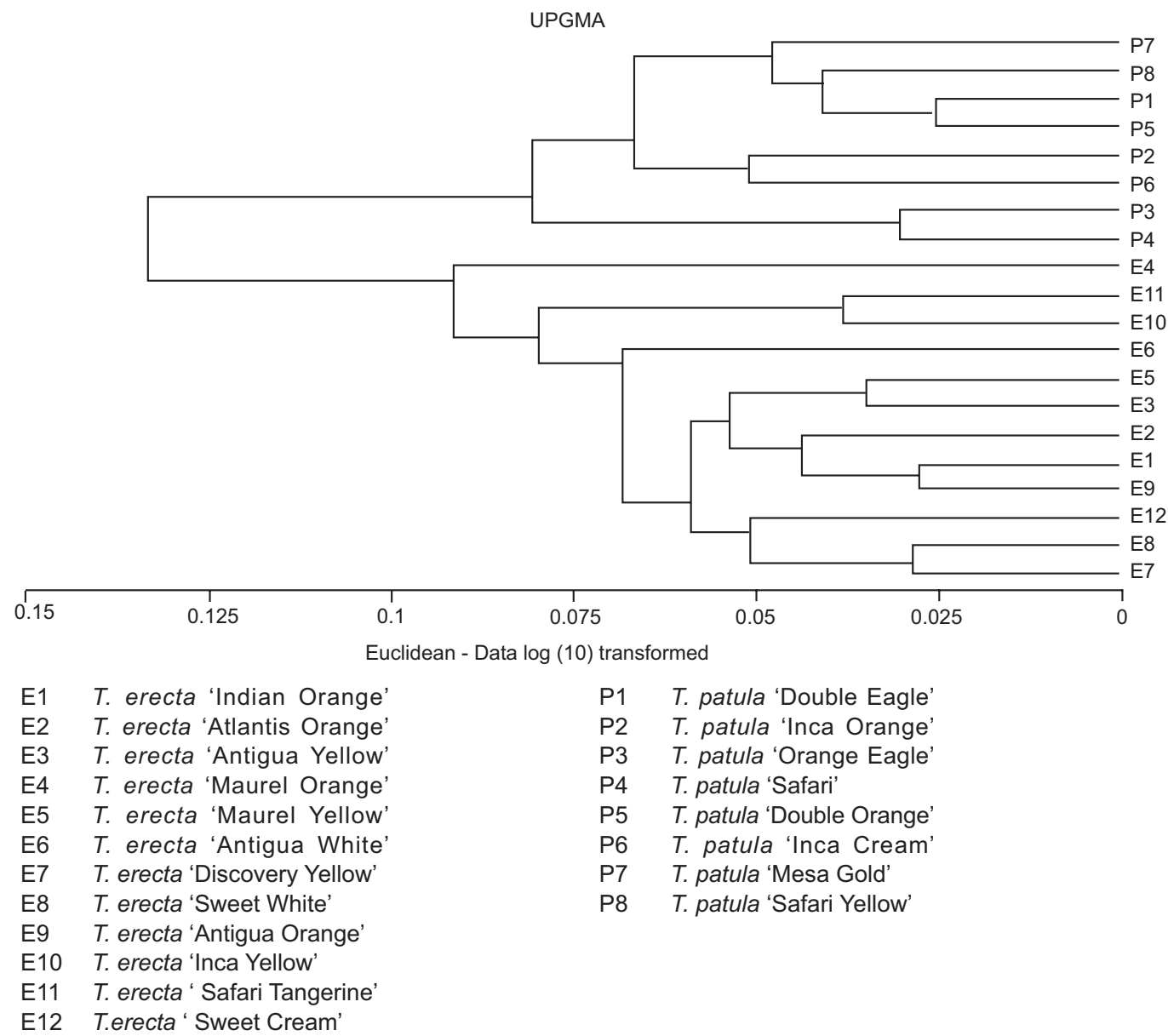

Fig. 1. UPGMA phenogram Tagetes based on morphological characters.

Table 4. PCA variable loading of morphology, (qualitative) in Tagetes cultivars

\begin{tabular}{|c|c|c|c|c|c|c|c|c|c|c|}
\hline \multirow[t]{2}{*}{ Anatomical features } & \multicolumn{5}{|c|}{ Tagetes erecta } & \multicolumn{5}{|c|}{ Tagetes patula } \\
\hline & Axis 1 & Axis 2 & Axis 3 & Axis 4 & Axis 5 & Axis 1 & Axis 2 & Axis 3 & Axis 4 & Axis 5 \\
\hline Stem surface & -0.058 & -0.470 & -0.458 & -0.112 & 0.560 & 0.077 & 0.397 & -0.466 & 0.209 & -0.115 \\
\hline Branching nature & -0.148 & -0.370 & 0.703 & 0.341 & 0.138 & 0.077 & 0.397 & -0.466 & 0.209 & -0.115 \\
\hline Petiole & 0.000 & 0.000 & 0.000 & 0.000 & 0.000 & -0.019 & -0.066 & -0.175 & 0.263 & 0.497 \\
\hline Leaf position & -0.070 & -0.382 & 0.274 & -0.265 & 0.059 & 0.378 & -0.048 & -0.041 & -0.088 & -0.465 \\
\hline Leaf Shape & 0.307 & -0.259 & -0.084 & -0.235 & 0.206 & 0.121 & 0.346 & -0.064 & -0.275 & 0.288 \\
\hline Leaf apex & 0.267 & -0.200 & -0.128 & -0.170 & -0.200 & -0.130 & 0.195 & 0.021 & -0.393 & 0.068 \\
\hline Leaf base & 0.196 & 0.046 & 0.184 & 0.166 & 0.521 & 0.247 & 0.029 & 0.246 & 0.444 & 0.507 \\
\hline Leaf margin & 0.000 & 0.000 & 0.000 & 0.000 & 0.000 & 0.000 & 0.000 & 0.000 & 0.000 & 0.000 \\
\hline Leaf colour & 0.000 & 0.000 & 0.000 & 0.000 & 0.000 & 0.000 & 0.000 & 0.000 & 0.000 & 0.000 \\
\hline Lamina symmetry & 0.000 & 0.000 & 0.000 & 0.000 & 0.000 & 0.000 & 0.000 & 0.000 & 0.000 & 0.000 \\
\hline Flower colour & 0.383 & -0.549 & -0.058 & 0.225 & -0.489 & 0.223 & 0.618 & 0.392 & -0.275 & 0.130 \\
\hline Head Type & 0.000 & 0.000 & 0.000 & 0.000 & 0.000 & 0.000 & 0.000 & 0.000 & 0.000 & 0.000 \\
\hline Inflorescence type & 0.000 & 0.000 & 0.000 & 0.000 & 0.000 & 0.000 & 0.000 & 0.000 & 0.000 & 0.000 \\
\hline Inflorescene position & 0.080 & 0.058 & 0.374 & -0.782 & -0.033 & 0.259 & -0.063 & 0.373 & -0.002 & -0.142 \\
\hline Involucre type & 0.000 & 0.000 & 0.000 & 0.000 & 0.000 & 0.000 & 0.000 & 0.000 & 0.000 & 0.000 \\
\hline Pappus type & 0.000 & 0.000 & 0.000 & 0.000 & 0.000 & 0.000 & 0.000 & 0.000 & 0.000 & 0.000 \\
\hline Style colour & 0.584 & 0.246 & 0.101 & 0.164 & 0.261 & 0.484 & -0.365 & -0.411 & -0.500 & 0.325 \\
\hline Stigma colour & 0.521 & 0.148 & 0.104 & -0.016 & -0.034 & 0.634 & -0.021 & 0.076 & 0.289 & -0.154 \\
\hline Stigma type & 0.000 & 0.000 & 0.000 & 0.000 & 0.000 & 0.000 & 0.000 & 0.000 & 0.000 & 0.000 \\
\hline Eigenvalues & 2.639 & 0.804 & 0.383 & 0.256 & 0.248 & 1.403 & 0.927 & 0.587 & 0.313 & 0.251 \\
\hline Percentage & 56.784 & 17.305 & 8.244 & 5.504 & 5.342 & 38.640 & 25.540 & 16.168 & 8.627 & 6.913 \\
\hline Cum. Percentage & 56.784 & 74.089 & 82.333 & 87.837 & 93.179 & 38.640 & 64.180 & 80.348 & 88.975 & 95.888 \\
\hline
\end{tabular}


Table 5. PCA variable loading of morphology, (quantitative) in Tagetes cultivars

\begin{tabular}{|c|c|c|c|c|c|c|}
\hline \multirow[t]{2}{*}{ Anatomical features } & \multicolumn{4}{|c|}{ Tagetes erecta } & \multicolumn{2}{|c|}{ Tagetes patula } \\
\hline & Axis 1 & Axis 2 & Axis 3 & Axis 4 & Axis 1 & Axis 2 \\
\hline Leaf length & -0.006 & 0.050 & -0.292 & -0.154 & -0.084 & 0.169 \\
\hline Leaf breadth & 0.649 & 0.590 & 0.403 & -0.052 & 0.004 & 0.648 \\
\hline Leaf area & -0.002 & 0.035 & 0.014 & 0.101 & 0.040 & 0.183 \\
\hline Leaf perimeter & -0.229 & 0.194 & 0.083 & 0.330 & 0.001 & -0.017 \\
\hline Petiole length & -0.240 & 0.219 & 0.185 & 0.561 & 0.085 & -0.050 \\
\hline Internode length & -0.515 & 0.017 & 0.679 & -0.401 & -0.344 & -0.139 \\
\hline Involucre length & -0.030 & -0.101 & -0.239 & 0.070 & 0.465 & 0.577 \\
\hline Pappus length & -0.440 & 0.692 & -0.373 & -0.154 & -0.396 & 0.138 \\
\hline Ovary length & -0.078 & -0.153 & 0.193 & 0.543 & 0.646 & -0.284 \\
\hline Style length & 0.057 & 0.222 & -0.128 & 0.235 & -0.275 & 0.253 \\
\hline Eigenvalues & 0.012 & 0.007 & 0.005 & 0.003 & 0.017 & 0.006 \\
\hline Percentage & 43.266 & 23.210 & 15.845 & 10.372 & 62.740 & 21.126 \\
\hline Cum. Percentage & 43.266 & 66.476 & 82.321 & 92.693 & 62.740 & 83.867 \\
\hline
\end{tabular}

and spongy parenchymatic tissue and in the cortical region of the petiole. The size of oil glands differed among the cultivars. In the cultivars of T. erecta the oil glands were larger in size compared with the oil glands in T. patula cultivars.

In the present study, an analysis of morphological characters of Tagetes species revealed that each cultivar is distinct. The two species of Tagetes (T. erecta and T. patula) used for this study were morphologically dissimilar. The shoot length of $T$. erecta ranged between 12 to $16 \mathrm{~cm}$, while the shoot length of $T$. patula ranged between 8 to $10 \mathrm{~cm}$ suggests that $T$. patula plants were smaller than T. erecta. Branching nature was also different. Most of the cultivars from T. patula were shown profusely branched nature. Variations could be noted in the flower colour. It was orange in T. erecta 'Indian orange'(E1), T. erecta 'Atlantis orange' (E2), T.erecta 'Maurel orange' (E4), T. erecta 'Antigua Orange' (E9) and T. erecta 'Safari Tangerine' (E11). Various yellowish shades were seen in T.erecta 'Antigua yellow' (E3), T. erecta 'Maurel yellow (E5), T. erecta' Discovery yellow' (E7) and T. erecta 'Inca Yellow' (E10). White, creamy white and cream colours were found in T. erecta 'Antigua white (E6), T. erecta 'Sweet white' (E8) and T. erecta' Sweet Cream' (E12) respectively. The most attractive flowers were observed in T. patula and head was heterogamous. The biggest flowerings form at $T$. erecta (Tagetes erecta 'Maurel Orange'[E4]) and smallest ones at T.patula (T.patula 'Inca Orange' [P2]).
Cluster analysis substantiated the existence of diversity among the 20 cultivars for the morphological traits studied. The clustering pattern showed that two principal clusters for two species, T. erecta 'Maurel orange' (E4) was distant from other cultivars of T. erecta. The dendrogram constructed on the basis of the data generated from the morphological traits of T. erecta also showed E4 was distinct from others. The colour of the flower was more attractive in this cultivar and other notable characters were the ribbed and hairy stem surface, long petiole, opposite leaves with serrate margin, leaf shape lanceolate and lamina symmetrical. Peduncle was found to be short $(5.5 \mathrm{~cm})$ compared to other cultivars. Biggest flowers with dark orange colour confirm its high pigment content rather than attractiveness. Anatomical characterization of leaf showed the presence of large oil glands in this cultivar. Both these characters make up this cultivar more economical.

Anatomical studies revealed that foliar secretory cavities were more in $T$. erecta cultivars than in T. patula cultivars. The size of oil glands differ in each cultivar. In the cultivars of $T$. erecta, the oil glands were larger in size compared with the oil glands in T. patula cultivars. From this present study it is clear that $T$. erecta cultivars are more appropriate to select as the parental genotype in the hybridization processes for obtaining different varieties and cultivars. Since many reports show that $T$. erecta are good source of yellow pigments and essential oil content useful for various activities (Verghese,1998). Ethnobotanical studies on $T$. erecta were also reported by many workers (Balick 
and Cox, 1996; Neher, 1968;). Vasudevan et al. (1997) has ranked T. erecta as a multipurpose herb.

A marigold flower contains abundant amount of a valuable antioxidant compound called lutein (Lavececchia et al.,2004). The extract with only the purified form with a lutein content of known concen-tration and a pure crystalline lutein isolated from marigold flower especially from $T$. erecta is allowed for food use. Dark coloured flowers contain about 200 times more lutein than the light coloured flowers. The concentration of lutein varies in different shades of marigold flowers, viz.; greenish yellow to bright yellow and orange brown (Gregory et al., 1986). The dark orange colour flowers are observed in T. erecta cultivars. From the present study it was evident that T. erecta 'Maurel orange' (E4) have large and dark orange coloured flowers.

Because of the wide range of activity marigolds are cultivated by farmers, but in India mostly for its yellow flowers. After harvesting they destroy the plants without utilizing its full benefits. Each and every part of the plant is very much useful. Simple distillation of the plant parts achieve good amount of essential oil which can be used for various purposes. Still the identification of superior cultivar is a difficult task. Various characters are spread in different cultivars and breeders can not find these characters in a single plant. Different genes which are distributed in different cultivars if pooled together in single plant will definitely create a superior plant which can be achieved through breeding. This study of preliminary screening of these cultivars by morphological and anatomical traits revealed that T. erecta cultivars are more appropriate to be selected as the parental genotype in the hybridization processes. Among this T.erecta 'Maurel orange' (E4) is superior in floral traits as well as large oil glands.

It is concluded from these results that different marker systems are appropriate to differentiate between the cultivars of Tagetes. Also, these marker systems could be complementary to each other and should be followed by molecular characterization using PCR-based markers to establish an integrated data about these cultivars. The maximum variability was present in flower colour. The cultivars with dark orange colour flowers can be used in further hybridization programme because of high carotenoid content. Selection of better cultivar can be made for species or varietal improvement on the basis of percent similarity with other species. Two more prominent characters, i.e., large oil glands and dark orange flowers can be chosen for the purpose.

\section{References}

Balick, J.M., Cox, P.A. 1996. Plants, people and culture: In: The Science of Ethnobotany. 228 pp. Scientific American Library, New York, USA.

Bukatsch, F. 1972. Bermerkungen zur Doppelfärbung Astrablau-Safranin. Mikrokosmos, 61: 255.

Charles, P.M., Li, T., Katherine, W.O. 2001. Analysis of carotenoid biosynthetic gene expression during marigold petal development. Plant Molecular Biology, 45: 281-293.

Cox, T.S., Murphy, J.P. 1990. The effect of parental divergence on F2 heterosis in winter wheat crosses. Theoretical and Applied Genetics, 79: 241-250.

Gregory, G.K., Chen, T.S., Philip, T. 1986. Quantitative analysis of lutein esters in marigold flowers by high performance liquid chromatography. Journal of Food Science, 51: 1093-1094.

Hickey, M., King, C. 2000. The Cambridge Illustrated Glossary of Botanical Terms. P. R. Shewry (ed.), vol. 35, 208 pp., Cambridge University Press, Cambridge, UK.

Lavececchia, C. 2004. Mediterranean diet and cancer. Public Health and Nutrition, 7: 965-968.

Neher, R.T.1968. The ethnobotany of Tagetes. Economic Botany, 22: 317-325.

Sneath, P.H.A., Sokal, R.R. 1973. Numerical taxonomy, In: The Principles and Practice of Numerical Classification, pp. 573-576, W.H. Freeman and Company, San Francisco, USA.

SPSS. 1999. Manuals SPSS Inc., Chicago, V-10.0.

Tian, H.Y., Wang, P., Shen, X.Q. 2007. Genetic analysis and botanical character in male sterile W205AB line of marigold. Northern Horticulture, 2: 105-107.

Trostle, R. 1968. The ethnobotany of Tagets Economic Botany, 22: 317-325.

Van Beuningen, L.T., Bush, R.H. 1997. Genetic diversity among North American spring wheat cultivars: I. Analysis of the coefficient of parentage matrix. Crop Science, 37: 570-579.

Vasudevan, P., Suman, K., Satyavathi, S. 1997. Tagetes: A multipurpose plant. Bioresource Technology, 62: 29-35.

Verghese, J. 1998. Focus on xanthophylls from Tagetes erecta L., the giant natural color complex-II. Indian Spices, 34: 13-16.

Wang, P. 2009. Selection and application of a new pigment Tagetes erecta 'Sesu 1'. Liaoning Agricultural Science, 2: 74-75.

Wang, G.Y. 2003. Technology on production of hybrid marigold seed in protected field. China Seed Industry, 10: 59-60.

Zhang, P., Zeng, L., Su, Y., Gong, X., Wang, X. 2011.Karyotype studies on Tagetes erecta L. and Tagetes patula L. African Journal of Biotechnology, 10: $16138-16144$. 